\title{
Coulisses
}

Revue de théâtre

40 | Hiver 2010

Jeux de rappels chez Marivaux

\section{La Surprise de l'amour (1727) : une comédie « seconde » de Marivaux}

Christelle Bahier-Porte

\section{(2) OpenEdition}

1 Journals

Édition électronique

URL : https://journals.openedition.org/coulisses/655

DOI : 10.4000/coulisses.655

ISSN : 2546-9460

Éditeur

Presses universitaires de Franche-Comté

\section{Édition imprimée}

Date de publication : 1 janvier 2010

Pagination : 43-58

ISBN : 978-2-84867-283-0

ISSN : $1150-594 X$

\section{Référence électronique}

Christelle Bahier-Porte, "La Surprise de l'amour (1727) : une comédie " seconde » de Marivaux», Coulisses [En ligne], 40 | Hiver 2010, mis en ligne le 30 novembre 2016, consulté le 29 décembre 2022. URL : http://journals.openedition.org/coulisses/655; DOI : https://doi.org/10.4000/coulisses.655

Ce document a été généré automatiquement le 29 décembre 2022.

Tous droits réservés 


\title{
La Surprise de l'amour (1727) : une comédie « seconde » de Marivaux
}

\author{
Christelle Bahier-Porte
}

$1 \quad$ La Surprise de l'amour est reçue par les Comédiens-Français le 30 janvier 1727. Le succès du Philosophe marié de Destouches (représenté à partir du 15 février) a conduit les comédiens à repousser la présentation de la pièce de Marivaux à la saison d'hiver. Pourtant, le 11 septembre, c'est L'T̂le de la raison qui est donnée au public du ThéâtreFrançais, pièce allégorique qui connaît un échec retentissant. Le Mercure de France annonce alors la représentation imminente de La Surprise au mois de novembre puis au mois de décembre ${ }^{1}$. La pièce est finalement représentée le mercredi 31 décembre. Ces faits nous semblent emblématiques de l'histoire complexe de La Surprise de 1727, comme si dès sa présentation elle se trouvait confrontée à la contrainte. La genèse de la pièce semble confirmer ce destin atypique. Le projet d'une " Nouvelle Surprise » aurait été annoncé dès le mois de mars $1726^{2}$ mais une brouille avec les Comédiens-Italiens aurait entravé le projet. Cette hypothèse n'est pas certaine mais si on l'admet, il faudrait alors comprendre que la «nouvelle » pièce n'était pas d'abord destinée au Théâtre-Français. Elle aurait donc été « déplacée » de l'Hôtel de Bourgogne vers la scène française. Rien ne permet de savoir en revanche si la pièce a été repensée et réécrite en fonction de ce déplacement.

2 Par ailleurs, la présence d'une dédicace en tête de la pièce, relativement rare chez Marivaux, confirme l'attention que l'auteur a portée à sa pièce, visant non la perfection mais une forme de «limite »: « il [l'auteur] a été aussi loin qu'il pouvait aller ». Les termes se rapportant à la "capacité » dans ce bref texte liminaire attirent de même l'attention sur le travail de l'auteur, voire sur sa « performance »: « rien de tout ce que j'étais capable de faire ", "ils observent avec finesse ce qu'il [l'auteur] est capable de faire " « elle [la pièce] avait à peu près le degré de bonté que je pouvais lui donner » ${ }^{3}$. Cette dédicace serait ainsi une réponse aux critiques (orales ou écrites) essuyées par la pièce qui ne connaît pas un grand succès : seuls les esprits « fins » peuvent apprécier la pièce ${ }^{4}$. 
3 Les critiques du XVIII ${ }^{\mathrm{e}}$ siècle s'attachent principalement à deux aspects : la comparaison avec la «première » Surprise de 1722 et la «nouveauté » du genre de la pièce. Dès décembre 1727, Le Mercure de France donne un long compte rendu précédé de quelques remarques sur la réception de la pièce «moins approuvée » que la Surprise italienne : " on convient que si quelque chose a contribué à en rendre le succès moins éclatant, c'est la nouveauté du genre ». La pièce s'est bien trouvée comme "déplacée " au Théâtre-Français où « on ne porte le même esprit » qu'au Théâtre-Italien. Le journaliste signale néanmoins que cette pièce "parfaitement bien écrite, pleine d'esprit et de sentiments» a reçu l'assentiment des "gens d'esprit» et "fins connaisseurs » ${ }^{5}$ Le Spectateur Littéraire publie en janvier 1728 une critique de la pièce en forme de conversation entre un "auteur à brochures" qui brocarde les défauts de la pièce (personnages et conduite de l'intrigue) et un chevalier qui défend le rôle d'Hortensius (fait rare !), loue l'esprit « solide » de la pièce et termine sur une allusion à la première Surprise : « je tiens un grand compte à $\mathrm{M}$ de $\mathrm{M}^{* * *}$ d'avoir osé risquer deux comédies sur un seul et même sentiment $»^{6}$. Le compte-rendu du Mercure de France mettait également en avant les « risques » encourus par les « premiers inventeurs » qui oseraient proposer un autre genre que celui de Molière ${ }^{7}$.

En mars 1728, Dominique et Romagnesi figurent, dans les premières scènes d'une pièce intitulée La Revue des théâtres, les deux Surprise de 1722 et 1727 sous les traits de deux sœurs, qui ont « la même conduite, les mêmes sentiments, à quelque chose près ». Par ses "manières à la mode » et sa " préciosité ", La Surprise française est sur le point de l'emporter mais l'arrivée d'Hortensius fait accorder la préférence à la pièce italienne ${ }^{8}$. Dès sa réception immédiate, la pièce est donc perçue à la fois comme "nouvelle ", sans que cette nouveauté ne soit clairement identifiée : nouveauté du genre par rapport à Molière, préciosité du langage ou "découvertes pour la morale moderne" - et " réchauffée » ${ }^{9}$ par rapport à son aînée italienne. La tension est inévitable parce qu'elle est tout simplement contenue dans le choix d'un titre identique pour les deux pièces, choix qui ne laisse pas d'être intrigant. Les critiques du xvIII siècle ne tentent pas de l'expliquer mais l'admettent et se livrent à l'inévitable comparaison.

5 Les critiques modernes, qui se sont d'ailleurs assez peu intéressés à cette pièce, proposent différentes hypothèses. Frédéric Deloffre et Françoise Rubellin dans l'édition du Théâtre complet écrivent : " Marivaux va au devant des critiques qu'on ne manquera pas de lui faire à propos de la ressemblance des sujets. Mieux, il affirme sa virtuosité souveraine en recourant au difficile procédé de la variation sur un thème connu. Tout le problème est alors de combler et de tromper l'attente du spectateur par un subtil mélange d'inédit et de déjà connu ${ }^{10}$. Henri Coulet et Michel Gilot notent également "l'audace " (qui fait écho aux "risques » signalés par les critiques du XvIII ${ }^{\mathrm{e}}$ siècle) de donner le même titre à deux œuvres et insistent sur la « confiance parfaite » de l'auteur « dans ses moyens d'expression et sa capacité d'étonner le public » ${ }^{11}$. C'est aussi parce que Marivaux choisit deux fois le même titre que l'on a pu faire de la "surprise de l'amour » une "structure matrice " de son théâtre. Pourquoi donc le choix d'un même titre? Pour le plaisir d'un exercice de style en proposant une Surprise à la Française après une Surprise à l'Italienne? Mais l'histoire de la pièce nous apprend que la «nouvelle Surprise» a pu être originellement destinée à la scène italienne. Art de la variation sur le même thème? Mais dans ce cas-là, d'autres pièces pourraient prétendre au titre de Surprise de l'amour. 
Sans prétendre apporter une réponse définitive, nous voudrions interroger ce choix à notre tour. Marivaux n'accomplit certes pas le geste de Pierre Ménard avec Don Quichotte, La Surprise de 1727 est bien différente de celle de 1722. Il accomplit pourtant un travail de déplacement, qui ne concerne pas seulement le lieu de représentation mais aussi l'« esprit », comme en témoignent les toujours précieux comptes rendus de l'époque. L'auteur s'impose ainsi une forme d'écriture à contraintes, dont l'histoire atypique de la représentation nous a semblé d'emblée emblématique. Il s'agit bien d'une gageure que de représenter une "autre " pièce sous le même titre. Si la pièce de 1727 peut être vue et lue sans référence à la première Surprise ${ }^{12}$, elle a néanmoins été pensée comme « seconde ».

7 En proposant une "seconde » Surprise de l'amour, Marivaux sollicite la mémoire du spectateur et propose un subtil travail de déplacement et de surenchérissement. Il nous semble que La Surprise de 1727 se fonde sur une dramaturgie de la contrainte qui confine au piège, pour les personnages comme pour les spectateurs; une contrainte que l'auteur s'impose pour rendre compte de son "audace» peut-être jusqu'à une certaine forme de limite (« aussi loin qu'il pouvait aller »). Mais Marivaux propose aussi une pièce qui invite à réfléchir sur le rapport entre amour (c'est bien le sujet de la pièce) et contrainte : peut-il y avoir une "seconde" surprise de l'amour pour des personnages qui ont déjà aimé ? Une " surprise » de l'amour - quel que soit le sens objectif ou réflexif que l'on donne à ce titre -, est-elle même seulement possible dans un monde de la contrainte et de la nécessité ? Paradoxalement, la «nouveauté » de $L a$ Surprise de l'amour de 1727 vient de sa position «seconde » qui lui permet toutes les audaces dramaturgiques et psychologiques jusqu'à la limite, jusqu'à ce que l'on se demande si ce fameux titre ne serait pas ironiquement antiphrastique.

\section{D'une « Surprise » à l'autre}

8 La Surprise de l'amour de 1727 n'est ni une suite ni véritablement une transposition «française » de la pièce italienne de 1722. Cependant, le choix d'un titre identique appelle la comparaison et invite notamment à étudier les variations dans la reprise de structures ou de personnages similaires, ce que nous nommions le travail de déplacement.

9 La situation initiale des deux pièces repose sur le refus d'aimer des deux personnages principaux : Lélio a été trahi par sa maîtresse infidèle, la Comtesse est veuve et méprise le sexe masculin sans doute après un mariage malheureux, semblable peut-être à ceux que peindra Silvia dans la première scène du Jeu de l'amour et du hasard. La Marquise est veuve, elle aussi, mais renonce à l'amour par fidélité à son époux avec lequel elle semble avoir partagé un amour exceptionnel. Le Chevalier a perdu, lui aussi, celle qu'il aimait : Angélique s'est réfugiée dans un couvent lorsque son père a refusé le mariage. Dans la pièce "italienne ", la "surprise de l'amour» peut se comprendre de deux manières: les personnages sont surpris par l'amour alors qu'ils pensaient ne plus pouvoir aimer, c'est le sens qui rapproche le plus les deux pièces. D'autre part, la Comtesse est surprise par l'amour car elle n'a jamais aimé ${ }^{13}$.

10 Cette interprétation est impossible dans la "seconde" pièce: la Marquise et le Chevalier ont aimé; la "surprise" de l'amour ne peut être une découverte du sentiment, ce qui change tout de même le sens de pièce : il ne s'agit pas d'aimer pour la première fois mais d'accepter d'aimer de nouveau. Nous nous demanderons d'ailleurs si 
cette «seconde » fois est réellement possible. Dans la première Surprise, la progression du sentiment chez les deux protagonistes se fait de manière parallèle et concomitante et se manifeste par des scènes symétriques de confidence des maîtres à leurs valets (par exemple : I, 6/II, 1 pour le refus d'aimer ; II, 5/III, 2 pour l'interprétation du billet). Il n'y a qu'un seul duo entre la Comtesse et Lélio à l'acte II, scène 7 au cours duquel les deux personnages tentent de persuader l'autre qu'il n'aime pas mais que l'autre aime. C'est dans cette scène unique que l'on trouve une référence à l'amitié en concurrence avec l'amour ${ }^{14}$, thème qui va être déplacé et amplifié dans la comédie "française ". L'amplification est d'ailleurs soulignée dans le texte : «je n'aurai jamais cru que l'amitié allât si loin, cela est surprenant ; l'amour est moins vif» (II, 9). Dans la première Surprise, il s'agit pour les deux protagonistes d'accepter de reconnaitre le sentiment amoureux, d'accepter de reconnaître que leur renonciation orgueilleuse à l'amour a été vaincue ${ }^{15}$. En revanche, dans La Surprise de 1727, ce qui est "surprenant », n'est pas le sentiment lui-même mais que l'on puisse aller «si loin » - c'est l'expression employée dans la dédicace pour qualifier la pièce - dans l'imposture.

Le déplacement et le surenchérissement dans la difficulté concernent également les personnages secondaires. En 1722, Colombine, puis Arlequin quand il aura accepté sa "conversion", ont pour mission d'aider les deux personnages à reconnaître ce sentiment qu'ils refusent. Les amours de Pierre et Jacqueline sont le prétexte des rencontres "forcées $»^{16}$ de Lélio et de la Comtesse et ne constituent nullement un obstacle à la progression du sentiment. En 1727, Lisette et Lubin semblent reprendre le rôle de Colombine et Arlequin mais le rapport aux maîtres est beaucoup plus complexe que dans la première pièce. Dans la première Surprise, maîtres et valets sont unis par une relation de confiance et d'affection signalée comme exceptionnelle ${ }^{17}$. Après qu'Arlequin a reconnu qu'il était amoureux de Colombine, les deux valets entendent travailler pour leurs maîtres certes mais aussi pour leur propre intérêt ${ }^{18}$.

On trouve un scénario similaire dans la seconde Surprise, ce qui relève autant de la reprise d'ailleurs que de la convention théâtrale. Lubin et Lisette entendent eux aussi favoriser leur union. Pourtant d'une pièce à l'autre, on pourra noter quelques "déplacements": c'est seulement à l'acte III scène 1 qu'Arlequin accepte de reconnaître explicitement qu'il aime Colombine et que les deux valets s'accordent pour " hâter l'amour de leurs maitres ». Certes, le valet est déjà " tracassé " par la beauté de la jeune suivante dès la scène I, 6 mais résiste héroïquement jusqu'au début de l'acte III. L'effet est comique car la sensualité, comme la gourmandise, est une des caractéristiques du type issu de la Commedia dell'arte : la résistance d'Arlequin crée un effet de discordance cocasse. Dans la pièce de 1727, la donne est différente : Lubin et Lisette se connaissent déjà (I, 1 : «c'est ce valet de campagne si naïf, qui vous a tant diverti il y a quelques jours»), Lubin "lorgne » Lisette dès la scène 3 et Lisette lui répond avec pragmatisme: «je n'ai que faire d'un homme qui part demain». La question se pose de nouveau à l'acte II, scène 1 : «à quoi nous servirait-il de nous aimer? ", Lubin suggère alors que leurs maîtres pourraient bien s'épouser par amitié, si on les y aide un peu. D'autre part, si Lubin retrouve quelques traits d'Arlequin, il ne s'agit plus de caractéristiques typiques dont Marivaux se jouerait mais de masques convoqués selon les besoins : Arlequin pleure volontiers avec son maître de la perte de leurs maîtresses et la résistance obstinée à sa sensualité gourmande est foncièrement comique. Lubin pleure lui aussi mais peut-être plus avec les larmes de Tartuffe qu'avec celles d'Arlequin, il s'agit moins de lazzi que de feinte ${ }^{19}$. 
13 La scène 11 de l'acte I est caractéristique de l'ambiguïté de Lubin dont la marque dramaturgique pourrait être l'identification de certaines répliques. Lorsque Lisette pose sans ambages les termes de la proposition : " Madame la Marquise est riche, jeune et belle ", Lubin commente : "cela est friand " et encourage son maître ("courage Monsieur ») sans qu'il soit précisé s'il s'agit d'apartés ou non. On comprend qu'il travaille surtout pour lui-même lorsque son maître réactive le souvenir d'Angélique : « Angélique me coupe la gorge » $(\mathrm{I}, 12)$; là encore rien ne précise s'il s'agit ou non d'un aparté. C'est Lubin qui raconte à Hortensius la proposition de Lisette au Chevalier (qui va déclencher l'indignation et le dépit de la Marquise) et prend l'initiative de parler à la Marquise de son maître (II, 4 « laisse-moi faire »), ce qu'il fait en II, 5 en demandant à la Marquise d'accepter le Chevalier pour qu'il puisse épouser Lisette, verbalisant, sans scrupule, ce que les valets de 1722 se disent secrètement. À l'acte III, il se réjouit de la tournure des événements et prend un malin plaisir à l'éviction des " rivaux ", comme s'il s'apprêtait à prendre à leur place le contrôle de la maison (comme Tartuffe) ${ }^{20}$.

Par contrepoint, le rôle de Lisette semble alors moins efficace que celui de son aînée Colombine. Certes, elle invite la Marquise à prendre conscience de ses « ressources » et à sortir de sa mélancolie dès la scène 1 (le premier duo entre la Comtesse et Colombine avait lieu en II, 1 dans la première Surprise). En revanche, tient-elle vraiment à favoriser l'amour entre le Chevalier et se maîtresse ? Son rôle est plus ambigu que celui de Lubin. Par exemple, on apprend à l'acte I scène 10 qu'elle a surpris la conversation entre la Marquise et le Chevalier. Qu'en a-t-elle compris? Elle dit au Chevalier qu'il ne doit pas entretenir la mélancolie de sa maîtresse et qu'il doit favoriser l'amour du Comte, ce qui suscite l'indignation du Chevalier qui se découvre un rival. Est-ce bonne foi de la part de Lisette ou stratagème ? Dès la scène suivante, elle propose en tout cas au Chevalier le cœur de sa maîtresse : parce qu'elle a compris par le « raisonnement surprenant » du Chevalier qu'il était amoureux d'elle (ce qu'elle dit en II, 6: "voilà ce qui m'avait fait parler moi ») ou parce qu'il convient de marier sa maîtresse à qui que ce soit, chevalier ou Comte (« dans l'état où je vois ma maîtresse, que m'importe par qui elle en sorte, pourvu qu'elle épouse un honnête homme» I,11). Dans ce cas, Lisette serait une incarnation dramaturgique de la contrainte mondaine, de l'obligation qu'a une femme d'être aimée ou en tout cas de ne pas être refusée, nous y reviendrons. Lorsque la Marquise découvre les intrigues de sa suivante, Lisette subit alors le même interrogatoire que Colombine dans la première Surprise (III, 2) : «Mon mariage avec le Comte, quand le terminerez-vous, Lisette? ", la suivante se défend alors en évoquant le dépit du Chevalier et l'assure en pleurant de son zèle et de son affection : "on n'a jamais aimé une maîtresse autant que je vous aime » (II, 6).

À la fin de la pièce, Lisette devient spectatrice, comme si elle assistait, impuissante, au déroulement contraint d'une action qui lui échappe. Au début de l'acte III, elle doit congédier Hortensius. Ce dernier croit comprendre ce qui se passe : «j'entends, c'est que la Marquise et le Chevalier ont de l'inclination l'un pour l'autre "; Lisette répond : "je n'en sais rien, ce ne sont pas mes affaires ", Lubin est plus direct : " on a un cœur, on s'en sert, cela est naturel ». Lisette doit encore congédier le Comte à la scène suivante, lequel n'est pas dupe et demande un entretien avec le Chevalier, c'est encore Lubin qui insiste sur l'amour qui unirait le Chevalier et la Marquise. Lisette ne revient qu'à la scène 12 pour entendre le désespoir de la Marquise, elle se contente d'abord de répéter ce que dit sa maîtresse en en tirant les conséquences : "renvoyez le Comte ", puis suggère que le « maudit » Chevalier serait « cause de tout cela »: «[...] et pour moi 
je crois que cet homme-là vous aime ", face à la dénégation de la Marquise elle conseille: "ne le revoyez plus», ce qui est interprété comme une nouvelle «persécution » par la Marquise. Lisette verbalise ici le dilemme de la Marquise plus qu'elle n'agit en faveur de l'un ou de l'autre des prétendants, ainsi à la scène suivante, c'est la Marquise elle-même qui prend la place de Lisette : " ne me conseilles-tu pas de le voir? Oui, n'est-ce pas?»; Lisette ne peut qu'acquiescer ironiquement: "oui, Madame, du ton que vous me le demandez, je vous le conseille» (III, 13). Lisette auraitelle perdu la conduite de l'action, si tant est qu'elle l'ait jamais menée ? Elle affirme en tout cas qu'elle "commence à comprendre" à la dernière scène. La fonction "machinatrice» semble donc s'être déplacée, d'une Surprise à l'autre, de la femme (Colombine) vers l'homme (Lubin) et l'affadissement du rôle de Lisette au cours de la pièce pourrait suggérer qu'elle se trouve elle aussi prise au piège, que l'intrigue lui a échappé et qu'elle se verra peut-être « contrainte » d'épouser Lubin.

Enfin, en 1727, la Marquise est confrontée aux avances du Comte et aux leçons d'Hortensius. Ce renforcement du rôle des personnages masculins par rapport à la première Surprise peut donner l'impression d'un harcèlement de la Marquise et crée un effet de focalisation sur ce personnage qui interroge la notion même de «surprise » de l'amour.

On cite souvent la définition donnée par Marivaux dans l'Avertissement des Serments indiscrets : « Dans La Surprise de l'amour, il s'agit de deux personnes qui s'aiment pendant toute la pièce, mais qui n'en savent rien eux-mêmes et qui n'ouvrent les yeux qu'à la dernière scène $»^{21}$. Mais de quelle Surprise s'agit-il dans cet Avertissement de 1732 ? La date et le lieu de représentation des Serments - la Comédie-Française - laissent à penser qu'il s'agit plutôt de la pièce de 1727. Et de fait, on pourrait appliquer la définition proposée à la seconde comédie ${ }^{22}$. Pourtant, la confusion est permise et la proposition est suffisamment générale pour désigner le canevas de base des deux pièces. La question se pose alors de savoir comment les personnages prennent conscience de ce sentiment et peut-être de manière plus insidieuse - et c'est ce que nous voudrions montrer pour la seconde Surprise - dans quelle mesure ce sentiment existe déjà ou est comme « forcé ».

La Comtesse et Lélio se trouvaient déjà confrontés en 1722 à une alternative d'abord posée par Arlequin : «être amoureux et ne l'être pas, ma foi, je donnerai le choix pour un liard. C'est misère : j'aime mieux la misère gaillarde que la misère triste » (SA, II, 5). De « misère », l'alternative devient «tyrannie » dans la bouche de Lélio (SA, III, 4) et se trouve de nouveau formulée par la Comtesse au moment du " dénouement » : « D'aimer ou de n'aimer pas ", ce à quoi Lélio répond : «Je vous reconnais : l'alternative est bien de vous, madame» (SA, III, 6). La réponse de la Comtesse est intrigante: «Eh! pas trop ». Reconnaissance, à demi-mot, que l'alternative n'en est pas une et qu'il s'agit bien d'aimer ou aveu, à demi-mot, que cette « alternative » n'est pas d'elle en effet mais qu'elle lui a été comme imposée ? Il nous semble que la « seconde » Surprise transforme l'alternative en piège et "ose interpréter $»^{23}$ le " pas trop » de la Comtesse en un «ce serait trop $»^{24}$.

\section{Une dramaturgie de la contrainte}

19 Revenons au cercle dont le Baron vient entourer les protagonistes de La Surprise de 1722. Nous avons pu montrer qu'il a une fonction symbolique et dramaturgique: il 
figure la « cérémonie » dans laquelle s'engagent les deux protagonistes qui refusent de reconnaître leur sentiment réciproque ${ }^{25}$. Mais comme l'a récemment souligné Karine Bénac, il figure aussi une forme d'enfermement qui oblige les personnages à ne pas déroger à leur rôle et à leur image, et engage le processus de l'amour-propre ${ }^{26}$. La "seconde " Surprise tirerait alors les conséquences dramaturgiques et psychologiques de ce «commerce forcé » que les protagonistes de la première Surprise disaient avoir ensemble. La pièce serait alors construite sur une logique implacable qui pousse jusqu'à ses limites (" aussi loin qu'il pouvait aller ») le principe de nécessité dramatique comme l'exploration des sentiments.

20 La Surprise de 1727 repose sur une double logique de la contrainte et de la nécessité. Tous semblent vouloir « forcer » la Marquise à aimer de nouveau. Mais cette contrainte externe est redoublée par une "nécessité » que s'imposent les personnages. Ces deux formes de contrainte font de la Surprise de 1727 une véritable machine infernale dont le personnage principal, sinon la victime, nous semble être la Marquise. À la différence de La Surprise de 1722 qui propose un cheminement parallèle des deux protagonistes, la pièce de 1727 se focalise en effet sur la Marquise. Elle est d'emblée offerte aux yeux des spectateurs, en tenue négligée, les cheveux épars ${ }^{27}$, comme surprise dans l'intimité de sa douleur. Au cours de la pièce, elle sera l'objet de toutes les attentions : Lisette veut la marier, Lubin veut la marier à son maître, le Comte veut l'épouser, Hortensius veut en garder la maîtrise et le Chevalier veut la garder pour lui seul également ; à tel point que l'on peut avoir l'impression que la Marquise est victime d'une forme de harcèlement, de complot visant à la faire céder. On notera par exemple, l'abondance des scènes collectives dans cette pièce et la rareté des duos. En effet, si le Chevalier et la Marquise se retrouvent cinq fois en duo (contre une seule fois pour Lélio et la Comtesse), quatre de ses duos sont en fait surpris ou espionnés : par Lisette (I, 7), par Hortensius (II, 7 et II, 9), par le Comte (III, 8). Seul le dernier duo (III, 15) semble véritable quoique rien ne soit dit du départ de Lubin et que le Comte en surprenne les « grands transports ».

21 L'effet de harcèlement ou de complot peut aussi être confirmé par la structure des actes. Au premier acte, la scène 7 marque un pivot qui pourrait d'ailleurs être un dénouement: la Marquise et le Chevalier, à la faveur de leur douleur commune, s'accordent une amitié qui leur tiendra lieu d'amour. La Marquise est alors absente de la deuxième partie de l'acte (qui comporte aussi 7 scènes) où se trament les différents complots dont elle est l'objet: le Chevalier formule tout d'abord sa satisfaction d'avoir trouvé une femme qui ressemble tant à son Angélique perdue, puis l'arrivée du Comte constitue une première péripétie avec une double issue possible : le Chevalier, en tant qu'ami, peut se faire l'adjuvant du Comte (I, 10) ou il peut lui même demander la Marquise en mariage (I, 11). Les deux rebondissements sont refusés par le Chevalier qui se réfugie dans le dépit et la colère ( $\mathrm{I}, 12)$. Lubin raconte - innocemment ? - à Hortensius ce qui vient de se produire et ce dernier promet de « soulever un orage qu'on ne pourra vaincre ».

L'acte II se focalise précisément sur les vains efforts d'Hortensius présent dans toutes les scènes (il est absent de la courte scène 2 mais Lisette ne parle que de lui et de son encombrant amour). Lorsque la Marquise apprend le double projet fomenté pour elle par sa suivante (II, 4), elle se présente comme victime "persécutée » et laisse transparaître quelque dépit quant au refus du Chevalier (II, 6). Le dialogue avec le Chevalier conduit à une forme de crise : il y est question d'" indifférence », de " haine " et de «dédain ». Après la scène comique de la colère du Chevalier contre le livre 
d'Hortensius, le Chevalier et la Marquise se promettent une amitié aussi exigeante que l'amour et décident de renvoyer Hortensius, bouc émissaire commode de leurs propres errements. On en revient donc au début de la pièce, à l'acte I scène 7 précisément. Cependant, le Comte est moins facile à congédier que le pédant Hortensius.

L'acte III est de nouveau construit en deux parties dont le pivot est la scène 8. Dans la première partie, le Comte œuvre pour lui-même à l'aide d'un "stratagème commun " qui pousse le Chevalier à nier son amour pour la Marquise et à accepter, pour preuve, le mariage avec la sœur du Comte. Le point culminant de ce qui relève bien d'une logique de la contrainte, du piège est atteint dans la scène 8 , scène à témoins cachés qui rappelle Britannicus (avec le Chevalier dans le rôle de Junie) : le Chevalier annonce qu'il en épouse une autre et la Marquise riposte en disant que le Comte ne lui déplaît pas. Par un nouvel effet de déplacement par rapport à la première Surprise, c'est le Comte, le rival, qui se retrouve aux genoux de la Marquise. À la scène 10, le piège semble scellé : un notaire doit venir « célébrer » une union : la Marquise devra qu'elle le veuille ou non se marier. Que ce soit finalement le Chevalier qui épouse la Marquise pourrait sembler une manière « heureuse » de clore la pièce, mais y avait-il une autre issue possible?

Cette contrainte externe qui s'impose à la Marquise pourrait être la manifestation dramaturgique de la contrainte sociale que la Marquise explicite à l'acte II scène $6^{28}$ et résume par la formule: «Je ne veux point me marier mais je ne veux pas qu'on me refuse ». Cependant, cela semble plus complexe, car cette nécessité externe est confirmée, renchérie par une forme de nécessité interne que s'imposent les personnages. Dès le début de la pièce, frappée par la douleur dont fait montre le Chevalier, la Marquise s'enferme dans une logique de l'exclusif et de l'exclusivité qui risque bien de sceller son destin: le Chevalier est présenté comme un "homme extraordinaire» qui «seul» peut la comprendre: «il n'y aura que moi qui vous plaindrai véritablement et vous êtes le seul qui rendra justice à mes pleurs », «je n'ai eu de moment supportable que celui-ci », «il n'y a qu'avec vous que ma douleur se verrait libre ».

25 A la fin de la scène, le Chevalier énonce explicitement le lien «nécessaire " qui les attache alors : «vous avez dans l'esprit une douceur qui m'était nécessaire, et qui me gagne » (I, 7). L'adjectif revient à la fin de l'acte II, lorsque la Marquise suggère que le Chevalier a rendu « nécessaire » sa colère après l'avoir refusée devant le Comte (II, 9). Cette nécessité conduit les personnages à se promettre une amitié jalouse, tout en refusant l'amour qui serait «trop ». La Marquise conclut alors : «Ecoutez, je n'avais pas moins besoin de cette explication-là que vous », et lorsque le Chevalier renchérit en affirmant: «je défie un amant de vous aimer plus que je fais », elle ajoute aussitôt: « cependant, il n'y a rien de trop ». De quoi a-t-elle «besoin » exactement? De savoir que l'amitié du Chevalier est exceptionnelle, exclusive - et donc un masque de l'amour qui ne se dit pas encore - ou qu'il s'agit bien seulement d'amitié et de rien de plus (qui serait « trop »)?

En tout état de cause, Marivaux explore avec une certaine ironie les conséquences dramaturgiques et psychologiques de cette logique étrange de l'exclusivité qui refuse l'amour. L'ironie est sensible notamment dans les répliques du Chevalier. Ainsi lorsqu'il apprend que la Marquise reçoit le Comte avec « douceur », il affirme : " je la distinguais, ce n'est qu'une femme comme une autre » et c'est Angélique qui reprend la première place : « s'il y a quelque chose au monde qui puisse me consoler, c'est de sentir combien vous êtes au-dessus de votre sexe, c'est de voir combien vous méritez mon amour» (I, 
12). Lorsque la Marquise lui avoue que le Comte est «presque le seul des amis » de son époux qu'elle reçoit, le Chevalier lui rétorque : «il est digne d'être excepté » et lui dit qu'il voit là une situation « fort ordinaire » (II, 7). À la scène suivante, c'est la Marquise qui est « exceptée » de la folie du monde : « cela va sans dire et c'est la règle » (II, 8). Il y a ici un jeu sémantique sur «exception » : être "excepté » c'est être "exceptionnel » ou bien être "omis", l'exclusivité contient aussi l'exclusion, ce dont Hortensius pourrait témoigner. Être "extraordinaire » ou n'être rien, cette logique exclusive conduit nécessairement à des situations limites : le Chevalier accepte d'en épouser une autre pour prouver que son amitié pour la Marquise n'est pas de l'amour: "mon attachement pour vous est trop délicat, pour profiter de l'honneur que cela me ferait " (III, 8). Quant à la Marquise, c'est paradoxalement une litote - « il ne m'a jamais déplu » - qui la conduit au mariage avec le Comte. Il est difficile en effet d'aller "aussi loin" dans la logique de la contrainte et on comprend que la pièce s'achève dans une atmosphère de folie : la Marquise croit perdre la raison, Lisette fait de la situation une « énigme » et le «bon sens » du Chevalier «s'en va » selon les termes de Lubin (III, 13). Comme Arlequin dans la première Surprise, Lubin fait un récit comique de la folie de son maître.

Comme le portrait glissé à l'insu de Lélio dans sa poche, le billet est lu à l'insu du Chevalier. C'est alors le billet, comme la cousine morte, qui permet l'aveu du mot interdit ( mon amour pour vous durera autant que ma vie») et la précipitation du dénouement. Si ce billet réactive la mémoire de la première Surprise, il renvoie aussi le spectateur au premier billet de la pièce, celui que le Chevalier destine à Angélique et fait lire à la Marquise (I, 7) et «déplace " alors de nouveau la problématique : de la conspiration plutôt bienveillante des valets dans la première Surprise à ce que Michel Gilot appelle une "comédie de l'aliénation ${ }^{29}$, voire à une possible manipulation des cœurs.

« Peut-on se rencontrer jusque là ! $(\mathrm{I}, 2)$ s'étonne la Marquise, « je n'aurais jamais cru que l'amitié allât si loin, cela est surprenant" confirme le Chevalier (II, 9). Avant Prévost, Marivaux explore donc les "états limites du sentiment»: la douleur hyperbolique qui demande compassion - comme celle de Cleveland quelque années plus tard - conduit la Marquise à élire un alter ego qui deviendra - à son corps défendant ? - un mari.

\section{Une comédie « seconde »}

C'est en effet à la fois la dramaturgie de la «surprise » et le sens de cette surprise qui est mis en question par Marivaux. Dans la comédie de 1727, les attentes des spectateurs sont comme "programmées" par la première pièce: ils savent que nécessairement ceux qui refusent d'aimer et se cachent derrière le masque de l'amitié finiront par être " surpris " par l'amour. Tout l'enjeu sera alors de "surprendre », malgré tout, le public et Marivaux choisit pour cela de faire de la contrainte et de la nécessité les enjeux même de la pièce : plutôt que de s'intéresser à l'avènement de cette surprise, ce qu'il pouvait faire dans la pièce de 1722, il va alors détourner l'attention du spectateur: l'objet du spectacle n'est plus la « surprise » de l'amour mais la négation même de cette surprise. La comédie « seconde » instaure alors un « nouveau genre » de plaisir théâtral qui en appelle à la mémoire du spectateur l'invitant au soupçon autant qu'à l'admiration. 
30 Nous avons vu plus haut que les personnages s'étonnent eux-mêmes de la singularité de leurs aventures. Le procédé est assez fréquent chez Marivaux, il permet d'instaurer une forme de connivence entre le dramaturge et le spectateur en soulignant un jeu avec les situations théâtrales convenues ou en prévenant d'éventuelles accusations d'invraisemblance. Dans la Surprise de 1727, la mémoire théâtrale du spectateur est par ailleurs sollicitée par les nombreuses références possibles à d'autres pièces du répertoire $^{30}$. Les contemporains de Marivaux ont noté la reprise du scénario de La Matrone d'Ephèse, remis à la mode par quelques pièces contemporaines.

31 La Marquise viendrait-elle grossir la liste des « jeunes veuves » éplorées ? Si on l'admet, la « surprise de l'amour » prend un tout autre sens et les atermoiements de la Marquise ne seraient que coquetterie. Marivaux se garde bien de faire explicitement de la Marquise un avatar de la matrone de Pétrone ou de la « jeune veuve » de La Fontaine, mais il n'empêche pas le rapprochement: la Marquise reçoit bel et bien deux prétendants, le Comte et le Chevalier et finit par accepter d'épouser l'un des deux (elle ne sait pas encore lequel à l'avant dernière scène) en une journée. Lubin, laisse d'ailleurs entendre au Chevalier que la situation de la Marquise est bien connue: «Mettez-vous à la place d'une veuve qui s'ennuie » et que la mémoire de son époux n'est qu'un vain prétexte : « qu'est-ce que vous voulez qu'elle fasse d'une mémoire » (I, 12). Que ce soit Lubin qui permette le soupçon est assez caractéristique du jeu « second » instauré : on peut le croire ou pas. Le Marquis d'Argenson notait cependant l'infléchissement de l'héritage : ce n'est pas par «tempérament » mais par le sentiment que la Marquise se laisserait séduire ${ }^{31}$.

32 Dans le progrès du sentiment, l'amitié joue un rôle essentiel. Déjà dans la première Surprise de 1722, la Comtesse et Lélio se proposaient d'être " amis » et de se divertir réciproquement: "vous à médire des femmes, moi à mépriser les hommes » (I, 7). L'amitié devient centrale dans la pièce de 1727. Le spectateur est alors invité à voir dans cette amitié un masque de l'amour qui refuse de se reconnaître, selon le schéma des «amours déguisés ${ }^{32}$. C'est la leçon de la première Surprise puisqu'à l'acte I scène 1 , l'«amiquié » est synonyme de l'amour, voire de la folie d'amour, pour les paysans Pierre et Jacqueline. On peut alors se demander dans quelle mesure les spectateurs sont dupes de ce qui ressemble si fort à une convention, un stratagème et se demander si le recours si insistant à l'amitié ne serait pas une forme non de consentement à l'amour mais de résistance car «l'amour ce serait trop ». Le topos des « amours contrariées » vient alors croiser le motif précédent.

33 Nous avons vu que les obstacles à l'amour ne sont pas seulement internes dans cette comédie. Comme le remarque, l'« auteur à brochures " mis en scène dans Le Spectateur littéraire de 1728, la pièce pouvait s'achever avant le début du deuxième acte, il suffisait que, en vertu du topos des "amours déguisés ", l'amitié qui unit le Chevalier et la Marquise soit identifiée comme de l'amour. Mais là est bien le problème. La grande réussite de Marivaux est alors de suggérer que les personnages qui tentent de faire obstacle à l'amour de la Marquise et du Chevalier ne sont peut-être que des " rivaux supposés »: Hortensius certes sème le trouble en rapportant les projets de Lisette mais il n'est qu'un « obstacle mou ${ }^{33}$ qui suscite des orages bien dérisoires et se voit évincé au début de l'acte III. Le Comte joue également un rôle étrange : il courtise la Marquise certes, mais il est aussi évincé au début de l'acte III et son recours à un «stratagème commun » pour faire avouer au Chevalier qu'il aime la Marquise finit par se retourner contre lui. La péripétie précipite en fait le dénouement attendu depuis le début, comme 
si tout était joué d'avance, sans surprise possible. On en vient alors à s'interroger sur le Chevalier lui-même, lui qui se trouve à l'origine comme à la fin de la folie exclusive de la Marquise : le «seul» qui puisse partager sa douleur devient le seul qu'elle doit épouser. Or, le jeu insidieux des échos intertextuels semble confirmer que l'amour est moins déguisé ou contrarié que «forcé ».

Rien n'interdit alors au spectateur/lecteur de relire/refaire la pièce en soupçonnant le Chevalier : il est ami du Marquis, parle comme lui et feint peut-être une douleur extrême ${ }^{34}$ pour mieux toucher la Marquise, "un caractère à peu près comme celui d'Angélique, et ce sont des trésors que ces caractères-là » (I, 8). Des « trésors » ... parce qu'ils sont faciles à séduire? Angélique fait penser à la religieuse portugaise regrettant fidèlement dans un couvent son libertin de séducteur. Frédéric Deloffre rappelle en effet qu'un "chevalier " au XVII ${ }^{e}$ et XVIII ${ }^{e}$ siècles, est un cadet de famille réduit à une « légitime » mais minime part d'héritage et ajoute «sa vocation véritable est celle d'un aventurier du sentiment $»^{35}$. Ce n'est qu'une lecture possible, un «tour» que peuvent prendre les choses, mais il faut bien avouer que la promesse faite à la Marquise (« Mon amour pour vous durera autant que ma vie ») reprenant littéralement celle faite à Angélique laisse à penser.

Le jeu avec la mémoire théâtrale du spectateur n'est pas propre à Marivaux et caractérise bon nombre de pièces de la première moitié du dix-huitième siècle. Les notices des éditions critiques de référence prouvent aussi que ce recours n'est pas propre à la seule Surprise de 1727. Il nous semble cependant que dans cette pièce qui se présente comme " seconde » en choisissant un titre identique à une pièce " première ", le procédé atteint une forme de limite qui le rend significatif. Les références ne sont pas seulement des échos intertextuels qui sollicitent la connivence amusée du public mais invitent le spectateur à s'interroger sur le sens profond de la pièce qui lui est donnée à voir. Il s'agit bien «d'admirer le tour que prennent les choses» (2SA, II, 6) quand la "surprise de l'amour" ne dépend plus de la "tournure grotesque de l'esprit de l'homme » (SA, I, 7) mais devient une nécessité, sociale et vitale et donc une illusion qui peut coûter la raison.

La Surprise de l'amour de 1727 est donc "seconde " à plus d'un titre. La comparaison s'impose avec l'aînée italienne mais les « déplacements » s'avèrent plus significatifs que les reprises et révèlent le parti-pris d'une dramaturgie qui fait de la contrainte son principe moteur. Nous avons bien affaire à une comédie « au second degré » qui use de la mémoire théâtrale du spectateur pour mieux mettre en question la dramaturgie comme la psychologie de «la surprise de l'amour». Nous n'avons pas voulu donner d'emblée un sens restreint à l'adjectif « seconde " pour pouvoir en explorer toutes les possibilités. La comédie de 1727 retrouve les principes poétiques des œuvres de jeunesse de l'auteur pour lesquelles Jean-Paul Sermain a mis en lumière les enjeux profonds d'une "position seconde» assumée ${ }^{36}$. Mais plus largement, avec toutes les précautions que demandent la hardiesse d'un tel rapprochement, la première et la seconde Surprise de l'amour entretiennent des liens de complémentarité et de dépassement comme la physique et la métaphysique, selon les commentateurs d'Aristote en quête de " philosophie première » et « philosophie seconde ». La physique de l'amour ${ }^{37}$ de la pièce première, se verrait questionnée par la méta-physique de la seconde. La pièce de 1727 s'essaye à problématiser, par la dramaturgie, ce que la critique théorisera ensuite comme un des principes structurels fondateurs, 
"premiers », de la dramaturgie et de la psychologie marivaudiennes: la surprise de l'amour.

\section{NOTES}

1. En novembre, le périodique annonce que les Comédiens-Français «doivent donner incessamment La Surprise de l'amour, comédie nouvelle en trois actes » et en décembre qu'ils "donneront incessamment la comédie nouvelle de La Surprise de l'amour », voir la Notice de La Seconde Surprise de l'amour, Théâtre complet, F. Deloffre et F. Rubellin [éd.], Classiques Garnier, 1989, t.I, p. 659.

2. La Surprise de l'amour de 1722 avait été rejouée à la Comédie-Italienne dès le $1^{\text {er }}$ janvier 1726 . Pour la genèse de la pièce voir la notice de H. Coulet et M. Gilot, Théâtre complet, La Pléiade, 19993, t. I, p. 1016.

3. "A son altesse sérénissime Madame la Duchesse du Maine ", La Surprise de l'amour - La Seconde Surprise de l'amour, H. Coulet [éd.], Folio Théâtre, [1993], 2005, p. 142-143. Toutes les références seront données dans cette édition.

4. La «finesse » de l'« esprit supérieur » qu'est la Duchesse est soulignée deux fois dans la dédicace.

5. L'intégralité du compte rendu est donnée dans l'édition citée du Théâtre complet, Ganier, t.I, p. 666-671.

6. Compte rendu cité par F. Rubellin et F. Deloffre, éd.citée, p. 662.

7. «Le genre que Molière a consacré au Théâtre-Français est le seul qu'on y cherche ; et s'il était possible qu'on y introduisît un meilleur, les premiers inventeurs risqueraient beaucoup ", Ibid., p. 666.

8. F. Rubellin donne les premières scènes de cette comédie dans son édition des deux Surprise, Le Livre de poche classique, 1991.

9. Selon le mot du Marquis d'Argenson, cité par F. Rubellin et F. Deloffre, éd.citée, p. 655.

10. Ibid., p. 656.

11. Marivaux, Théâtre complet, éd.citée, p. 1016.

12. Elle a d'ailleurs la préférence des dramaturges sur son aînée.

13. Voici comment elle définit l'amour : « [...] ces malheureuses situations si pleines de troubles, d'inquiétudes, de chagrins» (III, 2), La Surprise de l'amour, Folio Théâtre, p.123. Désormais abrégée en $S A$.

14. «[...] vous m'avez tantôt offert votre amitié, je ne vous demande que cela, je n'ai besoin que de cela » dit Lélio, SA, II, 7, p. 106.

15. Nous renvoyons sur ce point à notre article, C. Bahier-Porte, «Cette éternelle surprise de l'amour : aux origines du marivaudage ? », Coulisses- Revue de Théâtre, n 34, 2006, p. 123-138.

16. L'adjectif est employé par Lélio lorsqu'il dit à la Comtesse que le mariage de Pierre et Jacqueline est remis en cause : « cela finit le peu de commerce forcé que nous avons ensemble », SA, II, 7, p. 102.

17. Voir Arlequin : «Et moi, Monsieur, je vous assure que je vous aime cent fois plus aussi que de coutume, à cause que vous avez la bonté de m'aimer tant », SA, I, 2, p. 45 et Colombine : «je vous respecte et je vous aime vous le savez » III, 2, p. 122. 
18. «Ecoute, nous avons intérêt de hâter l'amour de nos maîtres, il faut qu'ils se marient ensemble », SA, III, 1, p. 114.

19. Voir le jeu sur le verbe «vouloir » dans la réplique explicite de Lubin : « moi je pleure parce que je le veux bien, car si je voulais je serais gaillard», 2SA, I, 3, p. 155.

20. Voir à ce propos l'emploi de «nous " dans son récit à Hortensius : « et si nous avions voulu d'elle, nous l'aurions eue par préférence, car Lisette nous l'a offert », 2SA, I, 14, p. 184.

21. Marivaux, Les Serments Indiscrets, "Avertissement ", Théâtre complet, La Pléiade, éd. citée, p. 662.

22. Ce que fait Michel Gilot dans la notice de la pièce, Ibid., p. 1178.

23. Au « pas trop » de la Comtesse, Lélio répond : «Pas trop... si j'osais interpréter ce mot-là ! », SA, III, 6, p. 137.

24. " Ah pour cela c'en serait trop; il ne faut pas, Chevalier, il ne faut pas » déclare la Marquise au Chevalier qui lui dit que son amitié pourrait bien devenir amour, 2SA, II, 9, p. 220.

25. C. Bahier-Porte, "Cette éternelle surprise de l'amour", aux origines du marivaudage, art. cité, p. 125-128.

26. K. Bénac, « De l'amour et de ses surprises dans La Surprise de l'amour, La Seconde Surprise de l'amour et Le Jeu de l'amour et du hasard», C. Ailloud-Nicolas [dir.], Marivaux, Les Préjugés vaincus?, PUF/CNED, 2009.

27. Voir la mise en scène de Luc Bondy.

28. Voir notamment la réplique sur « l'opinion » " qui nous donne tout, qui nous ôte tout », 2SA, p. 202-203.

29. Notice de La Seconde Surprise de l'amour, la Pléiade, éd. citée, p. 1023.

30. Voir les notices des deux éditions de référence citées pour un dossier complet sur les « sources » de la pièce.

31. "Le tempérament y fait ce que le sentiment fait dans notre pièce ", cité dans Théâtre complet, Garnier, éd.citée, p. 655.

32. Voir David Trott, «Des Amours déguisés à La Seconde Surprise de l'amour. Étude sur les avatars d'un lieu commun », Revue d'histoire littéraire de la France, mai-juin 1976, p. 373-384.

33. Selon l'expression de Jacques Scherer pour désigner Bartholo dans Le Mariage de Figaro, La Dramaturgie de Beaumarchais, Nizet, 1989.

34. Ce que suggère insidieusement Lubin en parlant de ses propres pleurs : « ils ont fait plaisir à Madame, et Monsieur le Chevalier l'accommodera bien autrement », 2SA, I, 3.

35. Marivaux, Théâtre complet, Garnier, éd.citée, p. 400.

36. Jean-Paul Sermain, Le Singe de Don Quichotte, Marivaux, Cervantès et le roman postcritique, Voltaire Foundation, Oxford, 1999.

37. Philippe Jousset étudie cet aspect «physique » de la pièce de 1722 dans un article intitulé, "Physique de Marivaux. Dramaturgie et langage dans La Surprise de l'amour», Revue Marivaux, $\mathrm{n}^{\circ} 5,1995$, p. 29-54.

\section{INDEX}

oeuvrecitee Surprise de l'amour (La) 


\section{AUTEURS}

CHRISTELLE BAHIER-PORTE

Université de Saint-Étienne 